\title{
EXPLICIT EIGENVALUES AND INVERSES OF TRIDIAGONAL TOEPLITZ MATRICES WITH FOUR PERTURBED CORNERS
}

\author{
WEN-CHYUAN YUEH ${ }^{\otimes 1}$ and SUI SUN CHENG ${ }^{2}$
}

(Received 5 February, 2007; revised 3 September, 2007)

\begin{abstract}
By means of a symbolic calculus for finding solutions of difference equations, we derive explicit eigenvalues, eigenvectors and inverses for tridiagonal Toeplitz matrices with four perturbed corners.
\end{abstract}

2000 Mathematics subject classification: primary 15A09, 15A18; secondary 39A10.

Keywords and phrases: Toeplitz matrix, eigenvalue, eigenvector, inverse, difference equation, sequence.

\section{Introduction}

Toeplitz matrices are intimately related to boundary value problems involving difference equations, and the corresponding relations have been exploited in $[2,3]$ for finding inverses of matrices arising from difference operators. In [8, 9], Yueh has found explicit formulae for the eigenvalues and eigenvectors as well as inverses when two 'corners' of the main diagonal of tridiagonal Toeplitz matrices are 'perturbed'. In this paper, explicit eigenvalues and eigenvectors as well as inverses for tridiagonal Toeplitz matrices with four 'perturbed corners' will be found. More specifically, we will consider the eigenvalue problem $A_{n} u=\lambda u$, where

$$
A_{n}=\left(\begin{array}{lllllll}
b+\gamma & c & 0 & 0 & \ldots & 0 & \alpha \\
a & b & c & 0 & \ldots & 0 & 0 \\
0 & a & b & c & \ldots & 0 & 0 \\
\ldots & \ldots & \ldots & \ldots & \ldots & \ldots & \ldots \\
\ldots & \ldots & \ldots & \ldots & \ldots & b & c \\
\beta & 0 & 0 & 0 & \ldots & a & b+\delta
\end{array}\right)_{n \times n}
$$

\footnotetext{
${ }^{1}$ Department of Refrigeration, Chin-Yi University of Technology, Taichung, Taiwan 411, Republic of China; e-mail: yuehwc@ncut.edu.tw.

${ }^{2}$ Department of Mathematics, Yuncheng University, Yuncheng, Shanxi 044000, People's Republic of China.

(C) Australian Mathematical Society 2008, Serial-fee code 0334-2700/08
} 
and $a, b, c, \alpha, \beta, \gamma, \delta$ are numbers in the complex plane $\mathbb{C}$. We will assume that $n \geq 3$ in the following discussions to avoid trivial cases.

We note that there are many algorithmic methods for finding eigenvalues and eigenvectors. Some of them may yield exact values but most of them either yield numerical values or 'unpleasant' formulae. Here, as in [8-10], we base our investigation on the method of symbolic calculus for difference equations in [1]. As we will see, we are able to derive a large number of exact eigenvalues and eigenvectors that appear to be more pleasant and can be used in other theoretical investigations. In the final section, we comment on alternate methods such as Trench's recursion or the Gohberg-Semencul formula that may yield unpleasant formulae, and we also justify our approach by several examples.

To prepare ourselves, we first recall some terminologies used in [1]. Let $\mathbb{N}$ denote the set of all nonnegative integers and let $l^{\mathbb{N}}$ be the set of complex sequences of the form $x=\left\{x_{k}\right\}_{k \in \mathbb{N}}$ endowed with the usual linear structure. A sequence of the form $\{\alpha, 0,0, \ldots\}$ is denoted by $\bar{\alpha}$ (or by $\alpha$ if no confusion is caused), and the sequence $\{0,1,0,0, \ldots\}$ is denoted by $\hbar$. Given two sequences $x=\left\{x_{k}\right\}$ and $y=\left\{y_{k}\right\}$ in $l^{\mathbb{N}}$, their convolution is denoted by $x * y$ (or $x y$ if no confusion is caused) and is defined by

$$
x y=\left\{\sum_{k=0}^{j} x_{k} y_{j-k}\right\}_{j \in \mathbb{N}} .
$$

Given two sequences $u=\left\{u_{k}\right\}$ and $v=\left\{v_{k}\right\}$ in $l^{\mathbb{N}}$ such that $u_{0} \neq 0$, the equation $x * u=v$ in the unknown sequence $x$ has a unique solution [1], which is denoted by $x=v / u$. It is easily verified that $\hbar^{2}=\hbar * \hbar=\{0,0,1,0,0, \ldots\}$ and $\hbar^{n}=\left\{\hbar_{j}^{n}\right\}_{j \in \mathbb{N}}$, $n=1,2, \ldots$, is given by $\hbar_{j}^{n}=1$ if $n=j$ and $\hbar_{j}^{n}=0$ otherwise. We will also set $\hbar^{0}=\overline{1}$.

Let $\lambda$ be an eigenvalue (which may be complex) and $\left(u_{1}, \ldots, u_{n}\right)^{\dagger}$ a corresponding eigenvector of $A_{n}$, where the dagger denotes transposition. Then $A_{n} u=\lambda u$ can be written as

$$
\begin{aligned}
b u_{1}+c u_{2} & =\lambda u_{1}-\left(\alpha u_{n}+\gamma u_{1}\right), \\
a u_{1}+b u_{2}+c u_{3} & =\lambda u_{2}, \\
\cdots & =\cdots, \\
a u_{n-2}+b u_{n-1}+c u_{n} & =\lambda u_{n-1}, \\
a u_{n-1}+b u_{n} & =\lambda u_{n}-\left(\beta u_{1}+\delta u_{n}\right),
\end{aligned}
$$

which is equivalent to the boundary value problem

$$
\begin{aligned}
a u_{k-1}+(b-\lambda) u_{k}+c u_{k+1} & =0, \quad k=1,2, \ldots, n, \\
a u_{0} & =\alpha u_{n}+\gamma u_{1}, \\
c u_{n+1} & =\beta u_{1}+\delta u_{n} .
\end{aligned}
$$


Note that if $c \neq 0$, then $u_{n}$ and $u_{1}$ cannot be 0 simultaneously, for otherwise from the first equation in (1.2), $u_{2}=0$. Inductively, we may then infer from the other equations that $u_{3}=u_{4}=\cdots=u_{n}=0$, which is contrary to the definition of an eigenvector. Similarly, by working backwards from the last equation in (1.2), if $a \neq 0$, then $u_{n}$ and $u_{1}$ cannot be 0 simultaneously.

If we view the numbers $u_{0}, u_{1}, u_{2}, \ldots, u_{n}$ and $u_{n+1}$, respectively, as the zeroth, first, second, $\ldots$, and the $(n+1)$ th term of an infinite (complex) sequence (we have used the same notation for a sequence and a vector that is regarded as its subsequence; this practice is convenient and will be used throughout) $u=\left\{u_{i}\right\}_{i=0}^{\infty}$, then the system of equations in (1.2) satisfies the three-term recurrence relation

$$
a u_{k-1}+b u_{k}+c u_{k+1}=\lambda u_{k}+f_{k}, \quad k=1,2, \ldots,
$$

under the condition

$$
a u_{0}=c u_{n+1}=0
$$

where $f=\left\{f_{k}\right\}_{k=0}^{\infty}$ is the infinite sequence defined by

$$
f_{k}= \begin{cases}-\left(\alpha u_{n}+\gamma u_{1}\right), & k=1, \\ -\left(\beta u_{1}+\delta u_{n}\right), & k=n \\ 0, & \text { otherwise }\end{cases}
$$

In sequence form, (1.3) can be expressed as

$$
c\left\{u_{k+2}\right\}_{k=0}^{\infty}+b\left\{u_{k+1}\right\}_{k=0}^{\infty}+a\left\{u_{k}\right\}_{k=0}^{\infty}=\lambda\left\{u_{k+1}\right\}_{k=0}^{\infty}+\left\{f_{k+1}\right\}_{k=0}^{\infty} .
$$

By taking the convolution of the above equation with $\hbar^{2}=\hbar \hbar$, and proceeding in a similar manner as in [10], we see that $u$ and $\lambda$ satisfy the condition

$$
\left(a \hbar^{2}+(b-\lambda) \hbar+\bar{c}\right) u=\left(c \overline{u_{1}}+f\right) \hbar .
$$

The above preparation is sufficient for us to find the formulae of the eigenvalues and the corresponding eigenvectors of $A_{n}$ for some special combinations of the numbers $a, b, c, \alpha, \beta, \gamma$ and $\delta$ in (1.1).

For the sake of convenience, the set of integers will be denoted by $\mathbb{Z}$. The number $\sqrt{-1}$ is denoted by $i$. We will also set

$$
\alpha \mathbb{Z}=\{m \alpha \mid m \in \mathbb{Z}\}, \quad \alpha \in \mathbb{C} .
$$

In particular, $\pi \mathbb{Z}$ denotes the set $\{\ldots,-2 \pi,-\pi, 0, \pi, 2 \pi, \ldots\}$.

\section{The case where $c=0$ and $a \neq 0$}

Suppose that $c=0, a \neq 0$ and $\alpha=0$ in (1.1). Then the matrix is triangular and the problem is trivial. So we consider only the case where $c=0, a \neq 0$ and $\alpha \neq 0$ in (1.1).

LEMma 2.1. Suppose that $c=0, a \neq 0$ and $\alpha \neq 0$. Then $b$ cannot be an eigenvalue of $A_{n}$. 
PROOF. If $c=0$, the first equation of (1.2) becomes

$$
(b+\gamma) u_{1}+\alpha u_{n}=\lambda u_{1} .
$$

If $b$ is an eigenvalue, then the second equation of (1.2) implies that $u_{1}=0$. But then (2.1) implies that $u_{n}=0$ if $\alpha \neq 0$. This violates our previous conclusion that $u_{1}$ and $u_{n}$ cannot be both zero when $a \neq 0$. This completes the proof.

Since $c=0$ and $b-\lambda \neq 0$, we may divide Equation (1.5) by $a \hbar+(b-\lambda)$ to obtain

$$
\begin{aligned}
u & =\frac{1}{a} \cdot \frac{f}{\hbar-\xi^{-1}} \\
& =\frac{-1}{a}\left\{\xi^{k+1}\right\} *\left\{0,-\left(\alpha u_{n}+\gamma u_{1}\right), 0, \ldots, 0,-\left(\beta u_{1}+\delta u_{n}\right), 0, \ldots\right\},
\end{aligned}
$$

where

$$
\xi=\frac{a}{\lambda-b} \neq 0
$$

The $j$ th term of $u$ is then

$$
u_{j}=\frac{1}{a}\left(\xi^{j}\left(\alpha u_{n}+\gamma u_{1}\right)+H_{j}^{n} \xi^{j+1-n}\left(\beta u_{1}+\delta u_{n}\right)\right), \quad j \geq 1,
$$

where $H_{j}^{n}$ is the unit step function defined by $H_{j}^{n}=1$ if $j \geq n$ and $H_{j}^{n}=0$ if $j<n$.

In particular,

$$
a u_{1}=\xi\left(\alpha u_{n}+\gamma u_{1}\right)
$$

and

$$
a u_{n}=\xi^{n}\left(\alpha u_{n}+\gamma u_{1}\right)+\xi\left(\beta u_{1}+\delta u_{n}\right)
$$

or, equivalently,

$$
(a-\xi \gamma) u_{1}-\xi \alpha u_{n}=0
$$

and

$$
\left(\xi \beta+\xi^{n} \gamma\right) u_{1}+\left(\xi^{n} \alpha+\xi \delta-a\right) u_{n}=0
$$

Since $u_{1}$ and $u_{n}$ cannot be both zero, we must necessarily have

$$
\left|\begin{array}{cc}
a-\xi \gamma & -\xi \alpha \\
\xi \beta+\xi^{n} \gamma & \xi^{n} \alpha+\xi \delta-a
\end{array}\right|=0
$$

or

$$
a \alpha \xi^{n}+(\alpha \beta-\gamma \delta) \xi^{2}+a(\gamma+\delta) \xi-a^{2}=0
$$

For each root $\xi$ of (2.6), we obtain from (2.2) a corresponding eigenvalue

$$
\lambda=b+\frac{a}{\xi} .
$$

A corresponding eigenvector $\left(u_{1}, \ldots, u_{n}\right)^{\dagger}$ may then be obtained from (2.3), (2.4) and (2.5), and this will be a nontrivial constant multiple of

$$
\left(1, \xi, \xi^{2}, \ldots, \xi^{n-2}, \frac{1}{\alpha \xi}(a-\gamma \xi)\right)^{\dagger}
$$


THEOREM 2.2. Suppose that $c=0, a \neq 0$ and $\alpha \neq 0$ in $A_{n}$. If $\lambda$ is an eigenvalue and $\left(u_{1}, \ldots, u_{n}\right)^{\dagger}$ a corresponding eigenvector, then $\lambda=b+a / \xi$, where $\xi$ is a root of Equation (2.6) and $\left(u_{1}, \ldots, u_{n}\right)^{\dagger}$ is a nontrivial constant multiple of the vector in (2.7).

There are some cases of interest.

Suppose that $\alpha \beta=\gamma \delta$ and $\gamma+\delta=0$. Then (2.6) is reduced to $\xi^{n}=a / \alpha$, and hence

$$
\xi_{k}=\left(\frac{a}{\alpha}\right)^{1 / n} \exp \left(i \frac{2 k \pi}{n}\right), \quad k=1,2, \ldots, n .
$$

If $\alpha=a$, then an eigenvector corresponding to

$$
\lambda_{k}=b+a \exp \left(-i \frac{2 k \pi}{n}\right)
$$

is a nontrivial constant multiple of

$$
\left(1, \exp \left(i \frac{2 k \pi}{n}\right), \exp \left(i \frac{4 k \pi}{n}\right), \ldots, \exp \left(i \frac{2(n-2) k \pi}{n}\right), \exp \left(-i \frac{2 k \pi}{n}\right)-\frac{\gamma}{a}\right)^{\dagger} .
$$

Since $\xi_{n}=1$, we see that there exists an eigenvalue $\lambda_{n}=b+a$, with the corresponding eigenvector being a nontrivial constant multiple of

$$
\left(1,1, \ldots, 1,1-\frac{\gamma}{a}\right)^{\dagger}
$$

In addition, if $\alpha=a$ and $n$ is even, then $\xi_{n / 2}=-1$, so there exists an eigenvalue $\lambda_{n / 2}=b-a$, with the corresponding eigenvector being a nontrivial constant multiple of

$$
\left(1,-1,1, \ldots, 1,-1-\frac{\gamma}{a}\right)^{\dagger}
$$

If $\alpha=-a$, then (2.6) is reduced to $\xi^{n}=-1$, and hence

$$
\xi_{k}=\exp \left(i \frac{(2 k-1) \pi}{n}\right), \quad k=1,2, \ldots, n,
$$

and an eigenvector corresponding to

$$
\lambda_{k}=b+a \exp \left(-i \frac{(2 k-1) \pi}{n}\right)
$$

is a nontrivial constant multiple of

$$
\left(1, \exp \left(i \frac{(2 k-1) \pi}{n}\right), \ldots, \exp \left(i \frac{(n-2)(2 k-1) \pi}{n}\right), \exp \left(-i \frac{(2 k-1) \pi}{n}\right)+\frac{\gamma}{a}\right)^{\dagger}
$$


If $\alpha=-a$ and $n$ is odd, then $\xi_{(n+1) / 2}=-1$, and hence there exists an eigenvalue $\lambda_{(n+1) / 2}=b-a$, with the corresponding eigenvector being a nontrivial constant multiple of

$$
\left(1,-1,1,-1, \ldots,-1,1+\frac{\gamma}{a}\right)^{\dagger}
$$

Furthermore, if $n=4 m+2, m=1,2, \ldots$, then $\xi_{m+1}=i$ and $\xi_{3 m+2}=-i$. Thus, there are two eigenvalues $\lambda_{m+1}=b-a i$ and $\lambda_{3 m+2}=b+a i$, with the corresponding eigenvectors being a nontrivial constant multiple of

$$
\left(1, i,-1,-i, \ldots, 1, i,-1,-i, 1, i+\frac{\gamma}{a}\right)^{\dagger}
$$

and

$$
\left(1,-i,-1, i, \ldots, 1,-i,-1, i, 1,-i+\frac{\gamma}{a}\right)^{\dagger}
$$

respectively.

We note that since our results are derived from the three-term recurrence relation (1.3), it is easily seen that for any eigenvalue $\lambda$, regardless of whether it is a simple root or a multiple root of (2.6), there is only one eigenvector (except for a constant multiple of it) belonging to this eigenvalue. For example, consider the matrix

$$
\left(\begin{array}{lll}
1 & 0 & 1 \\
1 & 1 & 0 \\
1 & 1 & 0
\end{array}\right),
$$

where $n=3, \alpha=\beta=1, \gamma=0, \delta=-1, a=b=1$. By (2.6) we have

$$
\xi^{3}+\xi^{2}-\xi-1=0 \quad \text { or }(\xi-1)^{2}(\xi+1)=0,
$$

and hence $\xi=1,1,-1$ and $\lambda=1+a / \xi=2,2,0$. For $\lambda=2$,

$$
u=\left(1, \xi, \frac{1}{\alpha \xi}(a-\gamma \xi)\right)=(1,1,1) .
$$

This is the only vector for the double-root eigenvalue $\lambda=2$ as may be checked by elementary methods.

The case where $a=0$ and $c \neq 0$ may be treated in a similar manner, or by applying the symmetry property of a matrix mentioned in the last paragraph of Section 3.1.

\section{The case where $a c \neq 0$}

Let $\lambda$ be an eigenvalue and let $\left(u_{1}, \ldots, u_{n}\right)^{\dagger}$ be a corresponding eigenvector of $A_{n}$ where $a c \neq 0$. Then $u=\left\{0, u_{1}, \ldots, u_{n}, \ldots\right\}$ satisfies (1.5). Since $c \neq 0$, we have from (1.5) that

$$
u=\frac{\left(c \overline{u_{1}}+f\right) \hbar}{a \hbar^{2}+(b-\lambda) \hbar+\bar{c}} .
$$


Let

$$
\gamma_{ \pm}=\frac{-(b-\lambda) \pm \sqrt{\omega}}{2 a}
$$

be the two roots of $a z^{2}+(b-\lambda) z+c=0$, where $\omega=(b-\lambda)^{2}-4 a c$. Since $\gamma_{+} \gamma_{-}=c / a \neq 0$, we may write

$$
\gamma_{ \pm}=\frac{1}{\rho} e^{ \pm i \theta}=\frac{1}{\rho}(\cos \theta \pm i \sin \theta)
$$

for some unique $\theta$ in the $\operatorname{strip}\{z \in \mathbb{C} \mid 0 \leq \operatorname{Re} z<\pi\}$, where $\rho=\sqrt{a / c}$. According to $\sin \theta=0$ or $\sin \theta \neq 0$, there are two cases to be considered:

Case I. Suppose that $\sin \theta \neq 0$ so that $\gamma_{+}$and $\gamma_{-}$are distinct. Then since

$$
\frac{1}{\rho} \cos \theta=\frac{\lambda-b}{2 a}
$$

and $a / \rho=c \rho$, we have (an error occurs in [8] by writing $\sqrt{a c}$ instead of $\rho c$ in formula (1.4) and all subsequent formulae; this error is corrected here)

$$
\lambda=b+2 c \rho \cos \theta
$$

and, by the method of partial fractions, we can then write (3.1) in the form $[1,8]$

$$
u=\frac{1}{\sqrt{\omega}}\left(\frac{1}{\gamma_{-}-\hbar}-\frac{1}{\gamma_{+}-\hbar}\right)\left(c \overline{u_{1}}+f\right) \hbar=\frac{2 i}{\sqrt{\omega}}\left\{\rho^{j} \sin j \theta\right\} *\left(c \overline{u_{1}}+f\right) .
$$

By evaluating the convolution product, we obtain the $j$ th term of $u$

$$
\begin{aligned}
u_{j}= & \frac{2 i}{\sqrt{\omega}}\left\{c u_{1} \rho^{j} \sin j \theta-\left(\alpha u_{n}+\gamma u_{1}\right) \rho^{j-1} \sin (j-1) \theta\right. \\
& \left.-H_{j}^{n}\left(\beta u_{1}+\delta u_{n}\right) \rho^{j-n} \sin (j-n) \theta\right\}
\end{aligned}
$$

for $j \geq 1$.

In particular,

$$
c \rho \sin \theta u_{n}=c u_{1} \rho^{n} \sin n \theta-\left(\alpha u_{n}+\gamma u_{1}\right) \rho^{n-1} \sin (n-1) \theta
$$

and

$$
0=c u_{1} \rho^{n+1} \sin (n+1) \theta-\left(\alpha u_{n}+\gamma u_{1}\right) \rho^{n} \sin n \theta-\left(\beta u_{1}+\delta u_{n}\right) \rho \sin \theta,
$$

where we have substituted $\sqrt{\omega}=2 i c \rho \sin \theta$ and $u_{n+1}=0$. These can be written as

$$
\begin{aligned}
& A u_{1}+B u_{n}=0, \\
& C u_{1}+D u_{n}=0,
\end{aligned}
$$


where

$$
\begin{aligned}
& A=\gamma \rho^{n-1} \sin (n-1) \theta-c \rho^{n} \sin n \theta, \\
& B=c \rho \sin \theta+\alpha \rho^{n-1} \sin (n-1) \theta \\
& C=\gamma \rho^{n} \sin n \theta+\beta \rho \sin \theta-c \rho^{n+1} \sin (n+1) \theta, \quad \text { and } \\
& D=\alpha \rho^{n} \sin n \theta+\delta \rho \sin \theta .
\end{aligned}
$$

Since $u_{1}$ and $u_{n}$ cannot both be zero, we must necessarily have

$$
\left|\begin{array}{ll}
A & B \\
C & D
\end{array}\right|=0
$$

This gives the necessary condition

$$
\begin{aligned}
& \rho^{n}(a c \sin (n+1) \theta+(\gamma \delta-\alpha \beta) \sin (n-1) \theta-c \rho(\gamma+\delta) \sin n \theta) \\
& \quad-\left(c \alpha \rho^{2 n}+a \beta\right) \sin \theta=0 .
\end{aligned}
$$

Once we have found $\theta$ that satisfies (3.10), we obtain an eigenvalue $\lambda$ by (3.2).

The eigenvectors may be found as follows. By (3.4), $A u_{1}=-B u_{n}$. Suppose that $B \neq 0$, then by substituting $u_{n}=-A u_{1} / B$ into (3.3),

$$
\begin{array}{r}
u_{j}=\frac{-u_{1}}{c \rho B} \rho^{j-1}\left(a c \sin j \theta+\alpha c \rho^{n} \sin (n-j) \theta-c \rho \gamma \sin (j-1) \theta\right), \\
j=1,2, \ldots, n .
\end{array}
$$

So we obtain an eigenvector that is a nontrivial constant multiple of the vector with components

$$
\begin{array}{r}
u_{j}=\rho^{j-1}\left(a \sin j \theta+\alpha \rho^{n} \sin (n-j) \theta-\gamma \rho \sin (j-1) \theta\right), \\
j=1,2, \ldots, n .
\end{array}
$$

Suppose that $A \neq 0$. Then, by substituting $u_{1}=-B u_{n} / A$ into (3.3), we may obtain the same result (3.11). On the other hand, by (3.5), $C u_{1}=-D u_{n}$. If $D \neq 0$, then, by substituting $u_{n}=-C u_{1} / D$ into (3.3),

$$
\begin{array}{r}
u_{j}=\frac{-u_{1}}{c \rho D} \rho^{j}\left(c \alpha \rho^{n} \sin (n+1-j) \theta+c \delta \rho \sin j \theta+(\alpha \beta-\gamma \delta) \sin (j-1) \theta\right), \\
j=1,2, \ldots, n .
\end{array}
$$

So we obtain an eigenvector that is a nontrivial constant multiple of the vector with components

$$
\begin{array}{r}
u_{j}=\rho^{j-1}\left(c \alpha \rho^{n} \sin (n+1-j) \theta+c \delta \rho \sin j \theta+(\alpha \beta-\gamma \delta) \sin (j-1) \theta\right), \\
j=1,2, \ldots, n .
\end{array}
$$


If $C \neq 0$, then, by substituting $u_{1}=-D u_{n} / C$ into (3.3), we obtain the same result (3.12).

Suppose that both $A=B=0$ and $C=D=0$. Then $u_{1}$ and $u_{n}$ may be arbitrary except for $u_{1}=u_{n}=0$. Let $u_{1}=0$ in (3.3). We have an eigenvector that is a nontrivial constant multiple of $u_{j}=\rho^{j-1} \sin (j-1) \theta$. Let $u_{n}=0$ in (3.3). We have an eigenvector that is a nontrivial constant multiple of

$$
u_{j}=c \rho^{j} \sin j \theta-\gamma \rho^{j-1} \sin (j-1) \theta .
$$

Hence in view of the linearity property of an eigenvector, we obtain an eigenvector with components

$$
u_{j}=c_{1} \rho^{j} \sin j \theta+c_{2} \rho^{j-1} \sin (j-1) \theta, \quad c_{1}, c_{2} \in \mathbb{C} .
$$

Case II. Suppose that $\sin \theta=0$, then we have $(b-\lambda)^{2}=4 a c$, and (3.1) can be written as

$$
u=\frac{1}{\tilde{\rho} c} \frac{\tilde{\rho} \hbar}{(\overline{1}-\tilde{\rho} \hbar)^{2}}\left(f+c \overline{u_{1}}\right)=\frac{1}{\tilde{\rho} c}\left\{j \tilde{\rho}^{j}\right\}_{j \in \mathbb{N}}\left(f+c \overline{u_{1}}\right),
$$

where

$$
\tilde{\rho}=\frac{\lambda-b}{2 c}= \pm \rho .
$$

The $j$ th term of $u$ for $j \geq 1$ now becomes

$$
u_{j}=\frac{1}{c \tilde{\rho}}\left\{c u_{1} j \tilde{\rho}^{j}-\left(\alpha u_{n}+\gamma u_{1}\right)(j-1) \tilde{\rho}^{j-1}-H_{j}^{n}\left(\beta u_{1}+\delta u_{n}\right)(j-n) \tilde{\rho}^{j-n}\right\} .
$$

In a similar manner, we obtain the necessary condition

$$
\tilde{\rho}^{n}((a c(n+1)+(\gamma \delta-\alpha \beta)(n-1))-\tilde{\rho} c(\gamma+\delta) n)-\left(\alpha c \tilde{\rho}^{2 n}+\beta a\right)=0 .
$$

Once we have found $\tilde{\rho}$ that satisfies $(3.15)$, we have by (3.14) that

$$
\lambda=b+2 \tilde{\rho} c .
$$

The eigenvectors may be found in a similar way. Let

$$
\begin{aligned}
\tilde{A} & =\gamma(n-1) \tilde{\rho}^{n-1}-c n \tilde{\rho}^{n}, \\
\tilde{B} & =c \tilde{\rho}+\alpha(n-1) \tilde{\rho}^{n-1} \\
\tilde{C} & =\gamma n \tilde{\rho}^{n}+\beta \tilde{\rho}-c(n+1) \tilde{\rho}^{n+1}, \quad \text { and } \\
\tilde{D} & =\alpha n \tilde{\rho}^{n}+\delta \tilde{\rho} .
\end{aligned}
$$

Suppose that $\tilde{A} \neq 0$ or $\tilde{B} \neq 0$. Then we may obtain an eigenvector that is a nontrivial constant multiple of the vector with components

$$
u_{j}=\tilde{\rho}^{j-1}\left(a j+\tilde{\rho}^{n} \alpha(n-j)-\tilde{\rho} \gamma(j-1)\right), \quad j=1,2, \ldots, n .
$$


Suppose that $\tilde{C} \neq 0$ or $\tilde{D} \neq 0$. Then we obtain an eigenvector that is a nontrivial constant multiple of the vector with components

$$
u_{j}=\tilde{\rho}^{j-1}\left(\tilde{\rho}^{n} c \alpha(n+1-j)+\tilde{\rho} c \delta j+(\alpha \beta-\gamma \delta)(j-1)\right), \quad j=1,2, \ldots, n .
$$

Suppose that both $\tilde{A}=\tilde{B}=0$ and $\tilde{C}=\tilde{D}=0$. Then an eigenvector is in the form

$$
u_{j}=c_{1} \tilde{\rho}^{j} j-c_{2} \tilde{\rho}^{j-1}(j-1) .
$$

THEOREM 3.1. Suppose that $a c \neq 0$ in the matrix $A_{n}$. Let $\lambda$ be an eigenvalue of the matrix $A_{n}$ and $u=\left(u_{1}, u_{2}, \ldots, u_{n}\right)^{\dagger}$ its corresponding eigenvector. If $\sin \theta \neq 0$ for some $\theta$ that satisfies (3.10), then the eigenvalues are given by $\lambda=b+2 c \rho \cos \theta$. The corresponding eigenvectors may be found by (3.11), (3.12) or (3.13). If $\sin \theta=0$ and (3.15) holds for some $\tilde{\rho}$, then the eigenvalues are given by $\lambda=b+2 \tilde{\rho} c$. The corresponding eigenvectors may be found by (3.18), (3.19) or (3.20).

Note that the necessary condition (3.10) or (3.15) is independent of $b$, and neither the formulae (3.11)-(3.13) nor the formulae (3.18)-(3.20) include the factor $b$. We see that when $a c \neq 0$, the eigenvectors of the matrix $A_{n}$ are independent of $b$.

Note also that when $\sin \theta=0$,

$$
\lambda=b+2 \tilde{\rho} c=b \pm 2 \rho c=b+2 \rho c \cos \theta, \quad \theta=0(\text { or } 2 \pi), \pi
$$

where $\theta=0$ (or $2 \pi$ ) corresponding to $\tilde{\rho}=+\rho$, and $\theta=\pi$ corresponding to $\tilde{\rho}=-\rho$.

Now we can apply the above results to find the eigenvalues and the corresponding eigenvectors of several matrices of the form (1.1) when $a c \neq 0$.

3.1. The case where $\boldsymbol{a}=\boldsymbol{c} \neq \mathbf{0}$ and $\boldsymbol{\alpha} \boldsymbol{\beta}-\boldsymbol{\gamma} \delta=\mathbf{0}$ For motivation, we first consider the case where $a=c \neq 0, \alpha \beta=\gamma \delta$ and $\alpha+\beta=\gamma+\delta=a$ in the matrix (1.1). Then the necessary condition (3.10) is reduced to $\sin (n+1) \theta-\sin n \theta-\sin \theta=0$ or

$$
\sin \frac{n \theta}{2} \sin \left(\frac{n+1}{2}\right) \theta=0, \quad \theta \notin \pi \mathbb{Z} .
$$

Consequently, $\sin n \theta / 2=0$ or $\sin (n+1 / 2) \theta=0$ for some $\theta \notin \pi \mathbb{Z}$. In the case when $\sin n \theta / 2=0$, we have $\theta=2 k \pi / n, \theta \notin \pi \mathbb{Z}$ so that an eigenvalue must be of the form

$$
\lambda_{k}=b+2 a \cos \frac{2 k \pi}{n}, \quad k=1,2, \ldots,\left[\frac{n-1}{2}\right],
$$

where $[x]$ denotes the integral part of $x$. In the case when $\sin (n+1 / 2) \theta=0$, we have $\theta=2 m \pi /(n+1), \theta \notin \pi \mathbb{Z}$, so that an eigenvalue must be of the form

$$
\lambda_{m+[(n-1) / 2]}=b+2 a \cos \frac{2 m \pi}{n+1}, \quad m=1,2, \ldots,\left[\frac{n}{2}\right] .
$$

On the other hand, since $\tilde{\rho}= \pm \rho= \pm 1$, the necessary condition (3.15) is reduced to

$$
\tilde{\rho}^{n}(n+1-\tilde{\rho} n)-1=0 .
$$


This can only be valid when $\tilde{\rho}=+1$, and by (3.16) we have $\lambda=b+2 a$ $=b+2 a \cos 0$. Thus $\theta=0$ is added to (3.22). Hence (3.22) is modified to

$$
\lambda_{m+[n-1 / 2]}=b+2 a \cos \frac{2(m-1) \pi}{n+1}, \quad m=1,2, \ldots,\left[\frac{n}{2}+1\right] .
$$

Note that we have $[(n-1) / 2]+[(n / 2)+1]=n$. Alternatively, (3.21) and (3.22) may be combined as

$$
\lambda_{k}= \begin{cases}b+2 a \cos \frac{2 k \pi}{n}, & k=1,2, \ldots,\left[\frac{n-1}{2}\right], \\ b+2 a \cos \frac{2(k-[(n-1) / 2]-1) \pi}{n+1}, & k=\left[\frac{n-1}{2}\right]+1, \ldots, n .\end{cases}
$$

In a similar manner we may find the eigenvalues for several other cases when $\alpha \beta=\gamma \delta$. We summarize them in the following theorem.

Theorem 3.2. Suppose that $a=c \neq 0$ and $\alpha \beta=\gamma \delta$ in the matrix (1.1). Then for various conditions of $\alpha+\beta$ and $\gamma+\delta$, the eigenvalues of $A_{n}$ are given by $\lambda_{k}=b+2 a \cos \theta_{k}$, where $\theta_{k}$ are given by:

(i) if $\alpha+\beta=\gamma+\delta=a$, then

$$
\theta_{k}= \begin{cases}2 k \pi / n, & k=1,2, \ldots,\left[\frac{n-1}{2}\right], \\ 2\left(k-\left[\frac{n-1}{2}\right]-1\right) \pi /(n+1), & k=\left[\frac{n-1}{2}\right]+1, \ldots, n ;\end{cases}
$$

(ii) if $\alpha+\beta=\gamma+\delta=-a$, then

$$
\theta_{k}= \begin{cases}(2 k-1) \pi / n, & k=1,2, \ldots,\left[\frac{n}{2}\right], \\ 2\left(k-\left[\frac{n}{2}\right]\right) \pi /(n+1), & k=\left[\frac{n}{2}\right]+1, \ldots, n ;\end{cases}
$$

(iii) if $\alpha+\beta=a$ and $\gamma+\delta=-a$, then

$$
\theta_{k}= \begin{cases}2 k \pi / n, & k=1,2, \ldots,\left[\frac{n-1}{2}\right], \\ \left(2\left(k-\left[\frac{n-1}{2}\right]\right)-1\right) \pi /(n+1), & k=\left[\frac{n-1}{2}\right]+1, \ldots, n ;\end{cases}
$$

(iv) if $\alpha+\beta=-a$ and $\gamma+\delta=a$, then

$$
\theta_{k}= \begin{cases}(2 k-1) \pi / n, & k=1,2, \ldots,\left[\frac{n}{2}\right], \\ \left(2\left(k-\left[\frac{n}{2}\right]\right)-1\right) \pi /(n+1), & k=\left[\frac{n}{2}\right]+1, \ldots, n ;\end{cases}
$$


(v) if $\alpha+\beta=\gamma+\delta=0$, then

$$
\theta_{k}=\frac{k \pi}{n+1}, \quad k=1,2, \ldots n ;
$$

(vi) if $\alpha+\beta=a$ and $\gamma+\delta=0$, then

$$
\theta_{k}= \begin{cases}2 k \pi / n, & k=1,2, \ldots,\left[\frac{n-1}{2}\right], \\ (2 k-1) \pi /(n+2), & k=\left[\frac{n-1}{2}\right]+1, \ldots, n ;\end{cases}
$$

(vii) if $\alpha+\beta=-a$ and $\gamma+\delta=0$, then

$$
\theta_{k}= \begin{cases}(2 k-1) \pi / n, & k=1,2, \ldots,\left[\frac{n}{2}\right], \\ 2 k \pi /(n+2), & k=\left[\frac{n}{2}\right]+1, \ldots, n\end{cases}
$$

(viii) if $\alpha+\beta=0$ and $\gamma+\delta=a$, then

$$
\theta_{k}=\frac{(2 k-1) \pi}{2 n+1}, \quad k=1,2, \ldots, n ;
$$

(ix) if $\alpha+\beta=0$ and $\gamma+\delta=-a$, then

$$
\theta_{k}=\frac{2 k \pi}{2 n+1}, \quad k=1,2, \ldots, n .
$$

To find the eigenvectors, we first note that, as long as $\alpha=0$, we have $B \neq 0$ and $\tilde{B} \neq 0$ by (3.7) and (3.17). Suppose that $\alpha=\gamma=0$; if $\sin \theta \neq 0$, then by (3.11) we have an eigenvector that is a nontrivial constant multiple of the vector with components

$$
u_{j}=\sin j \theta, \quad j=1,2, \ldots, n, \theta \neq 0, \pi .
$$

If $\sin \theta=0$, then by (3.18) we have an eigenvector that is a nontrivial constant multiple of the vector with components

$$
u_{j}=( \pm 1)^{j-1} j, \quad j=1,2, \ldots, n
$$

where the $+\operatorname{sign}$ corresponds to $\theta=0$ and the - sign corresponds to $\theta=\pi$.

Suppose that $\alpha=0$ and $\gamma=a$. If $\sin \theta \neq 0$, then, since $B \neq 0$, by (3.11) we have $u_{j}=\sin j \theta-\sin (j-1) \theta$ or

$$
u_{j}=\cos \left(j-\frac{1}{2}\right) \theta, \quad j=1,2, \ldots, n, \theta \neq 0, \pi,
$$

while if $\sin \theta=0$, by (3.15) the necessary condition for $A_{n}$ to have an eigenvalue is

$$
\tilde{\rho}^{n}(n+1-\tilde{\rho} n)-\beta / a=0 .
$$


This can only be satisfied when $\tilde{\rho}=1$, which corresponds to $\lambda=b+2 a \cos 0$, and $\beta=a$. Since $\tilde{B} \neq 0$, by (3.18) an eigenvector corresponding to $\theta=0$ is then given by a nontrivial constant multiple of the vector with components

$$
u_{j}=1, \quad j=1,2, \ldots, n .
$$

Note that this may also be written as (3.23) if we allow it to include $\theta=0$.

Similarly, suppose that $\alpha=0$ and $\gamma=-a$. If $\sin \theta \neq 0$, then, since $B \neq 0$, by Equation (3.11) we have an eigenvector that is a nontrivial constant multiple of $u_{j}=\sin j \theta+\sin (j-1) \theta$ or

$$
u_{j}=\sin \left(j-\frac{1}{2}\right) \theta, \quad j=1,2, \ldots, n, \quad \theta \neq 0, \pi,
$$

while if $\sin \theta=0$, by (3.15) the condition is

$$
\tilde{\rho}^{n}(n+1+\tilde{\rho} n)-\beta / a=0 .
$$

This can only be satisfied when $\tilde{\rho}=-1$, which corresponds to $\lambda=b+2 a \cos \pi$, and $\beta=a$ when $n$ is even or $\beta=-a$ when $n$ is odd. In either case, since $\tilde{B} \neq 0$, a corresponding eigenvector is given by a nontrivial constant multiple of the vector with components

$$
u_{j}=(-1)^{j-1}, \quad j=1,2, \ldots, n .
$$

As before, this may also be written as (3.24) if we allow it to include $\theta=\pi$.

To find the eigenvectors when $\alpha \neq 0$, we may apply (3.11)-(3.13) or (3.18)-(3.20). But if we suppose further that $\alpha \beta=\gamma \delta=0$, then we may apply the symmetry property of the matrix when $a=c$ to obtain simpler formulae. Let

$$
R_{n}=\left(\begin{array}{lllll}
0 & 0 & 0 & \ldots & 1 \\
0 & 0 & 0 & \ldots & 0 \\
\ldots & \ldots & \ldots & \ldots & \ldots \\
0 & 1 & 0 & \ldots & 0 \\
1 & 0 & 0 & \ldots & 0
\end{array}\right)_{n \times n}
$$

be the 'reflection' operator. Then $R_{n}^{-1}=R_{n}$ and $R_{n}\left(u_{1}, \ldots, u_{n}\right)^{\dagger}=\left(u_{n}, \ldots, u_{1}\right)^{\dagger}$, which is $\left(u_{1}, \ldots, u_{n}\right)^{\dagger}$ in reversed order. On the other hand, in the case when $a=c$ in $A_{n}$, let $A_{n}$ be abbreviated by

$$
\left(\begin{array}{lll}
\gamma & & \alpha \\
\beta & \searrow & \\
\beta & & \delta
\end{array}\right)
$$

Then

$$
R_{n}^{-1} A_{n} R_{n}=\left(\begin{array}{lll}
\delta & & \beta \\
& \searrow & \\
\alpha & & \gamma
\end{array}\right)
$$


Let $\lambda$ be an eigenvalue and $v=\left(v_{1}, \ldots, v_{n}\right)^{\dagger}$ be a corresponding eigenvector of $B_{n}=R_{n}^{-1} A_{n} R_{n}$, so we have $B_{n} v=\lambda v$. Then $R_{n} B_{n} v=\lambda R_{n} v$ implies that $A_{n} R_{n} v=\lambda R_{n} v$. Hence $\lambda$ is an eigenvalue and $R_{n} v=\left(v_{n}, \ldots, v_{1}\right)^{\dagger}$ is a corresponding eigenvector of $A_{n}$. Suppose that $\alpha \beta=0$ and $\alpha \neq 0$. Then $\beta=0$ in (3.25). This suggests an algorithm to find the eigenvectors of $A_{n}$ when $\alpha \neq 0$. Using the skill described above to find the eigenvectors $\left(v_{1}, \ldots, v_{n}\right)^{\dagger}$ of (3.25), the eigenvectors of $A_{n}$ are $\left(v_{1}, \ldots, v_{n}\right)^{\dagger}$ in reversed order, namely, $\left(u_{1}, \ldots, u_{n}\right)^{\dagger}$ $=\left(v_{n}, \ldots, v_{1}\right)^{\dagger}$. We summarize some of the results in the following theorem.

TheOrem 3.3. Suppose that $a=c \neq 0, \alpha \beta=\gamma \delta=0, \alpha+\beta= \pm a$ and $\gamma+\delta= \pm a$ in the matrix (1.1). Let $x^{(k)}=\left(x_{1}^{(k)}, x_{2}^{(k)}, \ldots, x_{n}^{(k)}\right)^{\dagger}, y^{(k)}=\left(y_{1}^{(k)}, y_{2}^{(k)}, \ldots, y_{n}^{(k)}\right)^{\dagger}$ and $z^{(k)}=\left(z_{1}^{(k)}, z_{2}^{(k)}, \ldots, z_{n}^{(k)}\right)^{\dagger}, k=1,2, \ldots, n$, where

and

$$
\begin{aligned}
& x_{j}^{(k)}=\left\{\begin{array}{ll}
\sin j \theta_{k}, & \theta_{k} \neq 0, \pi, \\
j, & \theta_{k}=0, \\
(-1)^{j-1} j, & \theta_{k}=\pi,
\end{array} \quad j=1,2, \ldots, n,\right. \\
& y_{j}^{(k)}=\cos \left(j-\frac{1}{2}\right) \theta_{k}, \quad \theta_{k} \neq \pi, \quad j=1,2, \ldots, n
\end{aligned}
$$

$$
z_{j}^{(k)}=\sin \left(j-\frac{1}{2}\right) \theta_{k}, \quad \theta_{k} \neq 0, \quad j=1,2, \ldots, n .
$$

Then the eigenvectors $u^{(k)}$ of $A_{n}$ corresponding to eigenvalue $\lambda_{k}$ in various conditions of $\alpha, \beta, \gamma$ and $\delta$ are given by

\begin{tabular}{|c|c|c|c|c|c|}
\hline & $\alpha$ & $\beta$ & $\gamma$ & $\delta$ & $u^{(k)}$ \\
\hline (i) & 0 & $*$ & 0 & $*$ & $x^{(k)}$ \\
\hline (ii) & 0 & $*$ & $a$ & 0 & $y^{(k)}$ \\
\hline (iii) & 0 & $*$ & $-a$ & 0 & $z^{(k)}$ \\
\hline (iv) & $*$ & 0 & 0 & $a$ & $R_{n} y^{(k)}$ \\
\hline (v) & $*$ & 0 & 0 & $-a$ & $R_{n} z^{(k)}$ \\
\hline (vi) & $*$ & 0 & $*$ & 0 & $R_{n} x^{(k)}$ \\
\hline
\end{tabular}

where the asterisk position may be either a or $-a$.

As an example, let

$$
A_{4}=\left(\begin{array}{llll}
4 & 3 & 0 & 3 \\
3 & 1 & 3 & 0 \\
0 & 3 & 1 & 3 \\
0 & 0 & 3 & 1
\end{array}\right)
$$

where $n=4, a=c=\alpha=\gamma=3, b=1, \beta=\delta=0$. Then $\alpha+\beta=\gamma+\delta=a$. By Theorem 3.2(i),

$$
\theta_{k}= \begin{cases}\pi / 2, & k=1 \\ 0,2 \pi / 5,4 \pi / 5, & k=2,3,4\end{cases}
$$


Hence the eigenvalues are

$$
\lambda_{1}=1, \quad \lambda_{2}=7, \quad \lambda_{3}=-\frac{1}{2}+\frac{3}{2} \sqrt{5}, \quad \lambda_{4}=-\frac{1}{2}-\frac{3}{2} \sqrt{5} .
$$

The eigenvectors given by Theorem 3.3(vi) are

$$
u_{j}^{(k)}=x_{4+1-j}^{(k)}=\left\{\begin{array}{ll}
\sin (4+1-j) \theta_{k}, & \theta_{k} \neq 0, \pi \\
(4+1-j), & \theta_{k}=0, \\
(-1)^{(4+1-j)}(4+1-j), & \theta_{k}=\pi
\end{array} \quad j, k=1,2,3,4\right.
$$

or

$$
\begin{gathered}
u_{j}^{(1)}=\sin \frac{(5-j) \pi}{2}, \quad u_{j}^{(2)}=5-j, \quad u_{j}^{(3)}=\sin \frac{2(5-j) \pi}{5} \text { and } \\
u_{j}^{(4)}=\sin \frac{4(5-j) \pi}{5} .
\end{gathered}
$$

Thus,

$$
\begin{aligned}
u^{(1)} & =(0,-1,0,1)^{\dagger} \\
u^{(2)} & =(4,3,2,1)^{\dagger} \\
u^{(3)} & =\left(\sin \frac{8 \pi}{5}, \sin \frac{6 \pi}{5}, \sin \frac{4 \pi}{5}, \sin \frac{2 \pi}{5}\right)^{\dagger} \approx\left(-1, \frac{1}{2}-\frac{1}{2} \sqrt{5},-\frac{1}{2}+\frac{1}{2} \sqrt{5}, 1\right)^{\dagger} \\
u^{(4)} & =\left(\sin \frac{16 \pi}{5}, \sin \frac{12 \pi}{5}, \sin \frac{8 \pi}{5}, \sin \frac{4 \pi}{5}\right)^{\dagger} \\
& \approx\left(-1, \frac{1}{2}+\frac{1}{2} \sqrt{5},-\frac{1}{2}-\frac{1}{2} \sqrt{5}, 1\right)^{\dagger} .
\end{aligned}
$$

3.2. The case where $a=c \neq 0$ and $\alpha \beta-\gamma \delta= \pm a^{2}$ The formulae obtained in the preceding subsection are consequences of Theorem 3.1 for special cases where $a c \neq 0$. Additional formulae may be found. They include formulae for special cases where $a c \neq 0$ and $\alpha=\beta=0$ (found in [8]), for some special cases where $a c \neq 0$ and $\gamma=\delta=0$ (found in [10]). Still more formulae for the cases where $a c \neq 0$ may be found, as long as Equation (3.10) could be simplified to obtain an explicit solution of some $\theta$ in the strip $\{z \in \mathbb{C} \mid 0 \leq \operatorname{Re} z<\pi\}$. For example, suppose that $a=c \neq 0, \gamma \delta-\alpha \beta=a^{2}$ and $\alpha+\beta=\gamma+\delta=0$. Then (3.10) is reduced to

$$
\sin (n+1) \theta+\sin (n-1) \theta=0
$$

or $\sin n \theta \cos \theta=0$. Consequently, $\sin n \theta=0$ or $\cos \theta=0$ for some $\theta \notin \pi \mathbb{Z}$. In the case when $\sin n \theta=0$, we have $\theta=k \pi / n, \theta \notin \pi \mathbb{Z}$ so that an eigenvalue must be of the form

$$
\lambda_{k}=b+2 a \cos \frac{k \pi}{n}, \quad k=1,2, \ldots, n-1 .
$$

In the case when $\cos \theta=0$, we have $\theta=\pi / 2$ and

$$
\lambda_{n}=b \text {. }
$$


Note that (3.15) is reduced to $(n+1)+(n-1)=2 n \neq 0$, and hence neither $\theta=0$ nor $\theta=\pi$ can be a solution in the present case.

As another example, suppose that $a=c \neq 0, \gamma \delta-\alpha \beta=a^{2}, \alpha+\beta=0$ and $\gamma+\delta=2 a$. Then (3.10) is reduced to $\sin (n+1) \theta+\sin (n-1) \theta-2 \sin n \theta=0$ or $\sin n \theta(\cos \theta-1)=0$. Since $\cos \theta \neq \pm 1$, we have $\theta=k \pi / n, \theta \notin \pi \mathbb{Z}$ so that an eigenvalue must be of the form

$$
\lambda_{k}=b+2 a \cos \frac{k \pi}{n}, \quad k=1,2, \ldots, n-1 .
$$

But now (3.15) is reduced to $(n+1)+(n-1)-\tilde{\rho} 2 n=0$, and this may be satisfied when $\tilde{\rho}=+1$, which corresponds to $\theta=0$; hence

$$
\lambda_{k}=b+2 a \cos \frac{(k-1) \pi}{n}, \quad k=1,2, \ldots, n .
$$

In a similar manner, we may find the eigenvalues for several other cases when $\alpha \beta-\gamma \delta= \pm a^{2}$. We summarize them in the following theorem.

THEOREM 3.4. Suppose that $a=c \neq 0$ and $\gamma \delta-\alpha \beta= \pm a^{2}$ in the matrix (1.1). Then for various conditions of $\alpha+\beta$ and $\gamma+\delta$, the eigenvalues of $A_{n}$ are given by $\lambda_{k}=b+2 a \cos \theta_{k}$, where $\theta_{k}$ are given by:

(i) if $\gamma \delta-\alpha \beta=a^{2}, \alpha+\beta=\gamma+\delta=0$, then

$$
\theta_{k}= \begin{cases}k \pi / n, & k=1,2, \ldots, n-1, \\ \pi / 2, & k=n ;\end{cases}
$$

(ii) if $\gamma \delta-\alpha \beta=a^{2}, \alpha+\beta=0$ and $\gamma+\delta=2 a$, then

$$
\theta_{k}=\frac{(k-1) \pi}{n}, \quad k=1,2, \ldots, n ;
$$

(iii) if $\gamma \delta-\alpha \beta=a^{2}, \alpha+\beta=0$ and $\gamma+\delta=-2 a$, then

$$
\theta_{k}=\frac{k \pi}{n}, \quad k=1,2, \ldots, n ;
$$

(iv) if $\gamma \delta-\alpha \beta=-a^{2}, \alpha+\beta=\gamma+\delta=0$, then

$$
\theta_{k}=\frac{(2 k-1) \pi}{2 n}, \quad k=1,2, \ldots, n ;
$$

(v) if $\gamma \delta-\alpha \beta=-a^{2}, \alpha+\beta=2 a, \gamma+\delta=0$, then

$$
\theta_{k}=\frac{2 k \pi}{n}, \quad k=1,2, \ldots, n ;
$$

(vi) if $\gamma \delta-\alpha \beta=-a^{2}, \alpha+\beta=-2 a, \gamma+\delta=0$, then

$$
\theta_{k}=\frac{(2 k-1) \pi}{n}, \quad k=1,2, \ldots, n
$$

We note that the corresponding eigenvectors may be found by (3.11)-(3.13) when $\sin \theta \neq 0$, and (3.18)-(3.20) when $\sin \theta=0$. 


\section{Necessary and sufficient conditions for the inverses}

The same method can be used to find the inverse of $A_{n}$. Let the (unique) inverse of $A_{n}$, if it exists, be denoted by

$$
G_{n}=\left(g^{(1)}\left|g^{(2)}\right| \ldots \mid g^{(n)}\right)=\left(\begin{array}{ccccc}
g_{1}^{(1)} & g_{1}^{(2)} & \ldots & \ldots & g_{1}^{(n)} \\
g_{2}^{(1)} & g_{2}^{(2)} & \ldots & \ldots & g_{2}^{(n)} \\
\ldots & \ldots & \ldots & \ldots & \ldots \\
g_{n}^{(1)} & g_{n}^{(2)} & \ldots & \ldots & g_{n}^{(n)}
\end{array}\right)_{n \times n} .
$$

Then $A_{n} G_{n}$ is the identity matrix $I_{n}$. By expanding $A_{n} G_{n}=I_{n}$, we obtain for each $k \in\{1, \ldots, n\}$,

$$
a g_{j-1}^{(k)}+b g_{j}^{(k)}+c g_{j+1}^{(k)}=\hbar_{j}^{k}+f_{j}^{(k)}, \quad j, k=1,2, \ldots, n,
$$

with $a g_{0}^{(k)}=c g_{n+1}^{(k)}=0$, where $f_{1}^{(k)}=-\left(\alpha g_{n}^{(k)}+\gamma g_{1}^{(k)}\right), f_{n}^{(k)}=-\left(\beta g_{1}^{(k)}+\delta g_{n}^{(k)}\right)$ and $f_{j}^{(k)}=0$ for $j=2, \ldots, n-1$. As in Section 1 , we may view the numbers $g_{1}^{(k)}, g_{2}^{(k)}, \ldots, g_{n}^{(k)}$ respectively as the first, second, .., and the $n$th term of an infinite (complex) sequence $g^{(k)}=\left\{g_{j}^{(k)}\right\}_{j \in \mathbb{N}}$ satisfying the three-term recurrence relation

$$
c\left\{g_{j+2}^{(k)}\right\}_{j \in \mathbb{N}}+b\left\{g_{j+1}^{(k)}\right\}_{j \in \mathbb{N}}+a\left\{g_{j}^{(k)}\right\}_{j \in \mathbb{N}}=\left\{\hbar_{j+1}^{k}\right\}_{j \in \mathbb{N}}+\left\{f_{j+1}^{(k)}\right\}_{j \in \mathbb{N}},
$$

and the condition $a g_{0}^{(k)}=c g_{n+1}^{(k)}=0$, where $f^{(k)}=\left\{f_{j}^{(k)}\right\}_{j \in \mathbb{N}}$ is the infinite sequence defined by

$$
f_{j}^{(k)}= \begin{cases}-\left(\alpha g_{n}^{(k)}+\gamma g_{1}^{(k)}\right), & j=1, \\ -\left(\beta g_{1}^{(k)}+\delta g_{n}^{(k)}\right), & j=n \\ 0, & \text { otherwise. }\end{cases}
$$

By the same procedure as in Section 1, we obtain

$$
\left(a \hbar^{2}+b \hbar+\bar{c}\right) g^{(k)}=\left(\bar{c} g_{1}^{(k)}+\hbar^{k}+f^{(k)}\right) \hbar .
$$

\section{The case where $c=0$ and $a b \neq 0$}

THEOREM 5.1. Suppose that $c=0$ and $a b \neq 0$ in matrix $A_{n}$. Then the inverse $G_{n}$ of $A_{n}$ given by (4.1) exists if, and only if,

$$
\Delta=b^{2}+b(\gamma+\delta)+(\gamma \delta-\alpha \beta)+b \alpha \psi^{n-1} \neq 0 .
$$

Furthermore, if $G_{n}$ exists, then

$$
g_{j}^{(1)}=\frac{1}{\Delta} \times \begin{cases}(b+\delta) \psi^{j-1}, & j<n, \\ b \psi^{n-1}-\beta, & j=n,\end{cases}
$$


and

$$
g_{j}^{(k)}=\frac{\psi^{j-k}}{\Delta} \times \begin{cases}-\alpha \psi^{n-1}, & j<k \\ -\alpha \psi^{n-1}+\Delta / b, & n>j \geq k \\ b+\gamma, & j=n\end{cases}
$$

for $2 \leq k \leq n$, where $\psi=-a / b$.

PROOF. In the case when $c=0$, since $b \neq 0$, we have by (4.3)

$$
g^{(k)}=\frac{1}{b+a \hbar}\left(\hbar^{k}+f^{(k)}\right)=\frac{1}{b}\left\{\psi^{j}\right\} *\left\{\hbar^{k}+f^{(k)}\right\}
$$

where $\psi=-a / b$. The $j$ th term of the sequence $g^{(k)}$ for $j \geq 1$ is

$$
g_{j}^{(k)}=\frac{1}{b}\left(-\left(\alpha g_{n}^{(k)}+\gamma g_{1}^{(k)}\right) \psi^{j-1}+H_{j}^{k} \psi^{j-k}-H_{j}^{n}\left(\beta g_{1}^{(k)}+\delta g_{n}^{(k)}\right) \psi^{j-n}\right) .
$$

Suppose that $k=1$ in $g_{j}^{(k)}$. By letting $j=1$ or $j=n$, we may obtain

$$
(b+\gamma) g_{1}^{(1)}+\alpha g_{n}^{(1)}=1
$$

and

$$
\left(\gamma \psi^{n-1}+\beta\right) g_{1}^{(1)}+\left(\alpha \psi^{n-1}+b+\delta\right) g_{n}^{(1)}=\psi^{n-1} .
$$

The necessary and sufficient condition for the unique $g^{(1)}$ to exist is that

$$
\Delta=\left|\begin{array}{cc}
b+\gamma & \alpha \\
\gamma \psi^{n-1}+\beta & \alpha \psi^{n-1}+b+\delta
\end{array}\right|=b^{2}+b(\gamma+\delta)+(\gamma \delta-\alpha \beta)+b \alpha \psi^{n-1} \neq 0 .
$$

Furthermore, if $g^{(1)}$ exists, then we have $g_{1}^{(1)}=\Delta_{1} / \Delta, g_{n}^{(1)}=\Delta_{n} / \Delta$, where

$$
\Delta_{1}=b+\delta, \quad \Delta_{n}=b \psi^{n-1}-\beta .
$$

Substituting these into (5.4), we obtain

$$
\begin{aligned}
g_{j}^{(1)}= & \frac{1}{b \Delta}\left\{-\alpha\left(b \psi^{n-1}-\beta\right) \psi^{j-1}-\gamma(b+\delta) \psi^{j-1}\right. \\
& +\left(b^{2}+b(\gamma+\delta)+(\gamma \delta-\alpha \beta)+b \alpha \psi^{n-1}\right) \psi^{j-1} \\
& \left.-H_{j}^{n}\left(\beta(b+\delta)+\delta\left(b \psi^{n-1}-\beta\right)\right) \psi^{j-n}\right\},
\end{aligned}
$$

which is (5.2).

Next suppose that $k>1$. We have by (5.4),

$$
(b+\gamma) g_{1}^{(k)}+\alpha g_{n}^{(k)}=0
$$

and

$$
\left(\gamma \psi^{n-1}+\beta\right) g_{1}^{(k)}+\left(\alpha \psi^{n-1}+b+\delta\right) g_{n}^{(k)}=\psi^{n-k}
$$


As above, the existence of a unique $g^{(k)}$ is equivalent to $\Delta \neq 0$. Furthermore, $\Delta_{1}$ and $\Delta_{n}$ are now given respectively by

$$
\Delta_{1}=-\alpha \psi^{n-k}, \quad \Delta_{n}=(b+\gamma) \psi^{n-k} .
$$

Substituting these and $g_{1}^{(k)}=\Delta_{1} / \Delta, g_{n}^{(k)}=\Delta_{n} / \Delta$ into (5.4), we obtain

$$
\begin{aligned}
g_{j}^{(k)}= & \frac{1}{b \Delta}\left\{-\alpha(b+\gamma) \psi^{n-k} \psi^{j-1}-\gamma\left(-\alpha \psi^{n-k}\right) \psi^{j-1}\right. \\
& +H_{j}^{k}\left(b^{2}+b(\gamma+\delta)+(\gamma \delta-\alpha \beta)+b \alpha \psi^{n-1}\right) \psi^{j-k} \\
& \left.-H_{j}^{n}\left(\beta\left(-\alpha \psi^{n-k}\right)+\delta(b+\gamma) \psi^{n-k}\right) \psi^{j-n}\right\},
\end{aligned}
$$

which is (5.3). The proof is complete.

As a special case, suppose that $\psi=-a / b=1$. Then if

$$
\Delta=b(b+\alpha+\gamma+\delta)+(\gamma \delta-\alpha \beta) \neq 0,
$$

then

$$
g_{j}^{(1)}=\frac{1}{\Delta} \times \begin{cases}b+\delta, & j<n, \\ b-\beta, & j=n,\end{cases}
$$

and, for $k=2, \ldots, n$,

$$
g_{j}^{(k)}=\frac{1}{\Delta} \times \begin{cases}-\alpha, & j<k, \\ -\alpha+\Delta / b, & n>j \geq k, \\ b+\gamma, & j=n\end{cases}
$$

As another special case, suppose that $\beta=\gamma=\delta=0$. Then if $\Delta=b^{2}+b \alpha \psi^{n-1} \neq 0$,

$$
g_{j}^{(k)}=\frac{\psi^{j-k}}{\Delta} \times \begin{cases}-\alpha \psi^{n-1}, & j<k, \\ b, & j \geq k,\end{cases}
$$

for $k=1,2, \ldots, n$. We note that this equation is also applicable when $\alpha=0$.

Suppose that $a=0$ and $b c \neq 0$ in $A_{n}$. Then the inverse may be found by using the equality $A^{-1}=\left(\left(A^{T}\right)^{-1}\right)^{T}$ and the formulae obtained in this section.

\section{The case where $a c \neq 0$}

THEOREM 6.1. Suppose that ac $\neq 0$ in matrix $A_{n}$. If $b^{2}-4 a c \neq 0$, then the inverse $G_{n}$ of $A_{n}$ given by (4.1) exists if, and only if,

$$
\begin{aligned}
\Delta= & \rho^{n} \sin \phi(a c \sin (n+1) \phi+(\gamma \delta-\alpha \beta) \sin (n-1) \phi-c \rho(\gamma+\delta) \sin n \phi) \\
& -\left(c \alpha \rho^{2 n}+a \beta\right) \sin ^{2} \phi \neq 0,
\end{aligned}
$$


where $\rho=\sqrt{a / c}$ and $\phi$ is the unique complex number in the strip $\{\phi \in \mathbb{C} \mid 0 \leq \operatorname{Re} \phi$ $<\pi\}$ such that $\cos \phi=-b / 2 c \rho$. Furthermore, if $G_{n}$ exists, then

$$
\begin{aligned}
g_{j}^{(k)}= & \frac{\rho^{n+j-k}}{\Delta} \times\left\{\sin (n-k) \phi\left(\delta \sin j \phi+\frac{\alpha \beta-\gamma \delta}{c \rho} \sin (j-1) \phi\right)\right. \\
& \left.+\sin (n+1-k)(\gamma \sin (j-1) \phi-c \rho \sin j \phi)+\alpha \rho^{n-1} \sin (j-k) \phi \sin \phi\right\}
\end{aligned}
$$

for $j<k$ and

$$
\begin{aligned}
g_{j}^{(k)}= & \frac{\rho^{n+j-k}}{\Delta} \times\left\{\sin (n-j) \phi\left(\delta \sin k \phi+\frac{\alpha \beta-\gamma \delta}{c \rho} \sin (k-1) \phi\right)\right. \\
& \left.+\sin (n+1-j)(\gamma \sin (k-1) \phi-c \rho \sin k \phi)+\beta \rho^{1-n} \sin (k-j) \phi \sin \phi\right\}
\end{aligned}
$$

for $j \geq k$. If $b^{2}-4 a c=0$, then the inverse exists if, and only if,

$$
\Delta=-\alpha c \rho^{2 n}-a \beta+\rho^{n}(a c(n+1)+(\gamma \delta-\alpha \beta)(n-1)-c \rho(\gamma+\delta) n) \neq 0,
$$

where $\rho=-b / 2 c$. Furthermore, if $G_{n}$ exists, then

$$
\begin{aligned}
g_{j}^{(k)}= & \frac{\rho^{n+j-k}}{\Delta}\left\{(n-k)\left(\delta j+\frac{(\alpha \beta-\gamma \delta)}{c \rho}(j-1)\right)\right. \\
& \left.+(n+1-k)(\gamma(j-1)-c \rho j)+\rho^{n-1} \alpha(j-k)\right\}
\end{aligned}
$$

for $j<k$ and

$$
\begin{aligned}
g_{j}^{(k)}= & \frac{\rho^{n+j-k}}{\Delta}\left\{(n-j)\left(\delta k+\frac{(\alpha \beta-\gamma \delta)}{c \rho}(k-1)\right)\right. \\
& \left.+(n+1-j)(\gamma(k-1)-c \rho k)+\rho^{1-n} \beta(k-j)\right\}
\end{aligned}
$$

for $j \geq k$.

ProOF. Since $c \neq 0$, we can divide (4.3) by $a \hbar^{2}+b \hbar+\bar{c}$ to obtain

$$
g^{(k)}=\frac{\left(\bar{c} g_{1}^{(k)}+\hbar^{k}+f^{(k)}\right) \hbar}{a \hbar^{2}+b \hbar+\bar{c}} .
$$

Let

$$
\eta_{ \pm}=\frac{-b \pm \sqrt{\omega}}{2 a}
$$

be the two roots of $a z^{2}+b z+c=0$, where $\omega=b^{2}-4 a c$. According to $\omega \neq 0$ or $\omega=0$, there are two cases to be considered. 
Case I. Suppose that $\omega \neq 0$ so that $\eta_{+}$and $\eta_{-}$are two different numbers. Since $\eta_{+} \eta_{-}=c / a \neq 0$, we may write $\eta_{ \pm}=e^{ \pm i \phi} / \rho$ for some unique $\phi$ in the strip $\{\phi \in \mathbb{C} \mid 0 \leq \operatorname{Re} \phi<\pi\}$, where $\rho=\sqrt{a / c}$ and $\cos \phi=-b / 2 \rho c$. In addition, since $\eta_{+} \neq \eta_{-}$, we also have that $\sin \phi \neq 0$ and $\cos \phi \neq \pm 1$.

By the method of partial fractions, we may write (6.7) in the form

$$
g^{(k)}=\frac{2 i}{\sqrt{\omega}}\left\{\rho^{j} \sin j \phi\right\}_{j=0}^{\infty} *\left(\bar{c} g_{1}^{(k)}+\hbar^{k}+f^{(k)}\right) .
$$

Then we have, by evaluating the convolution in the expression for $g^{(k)}$,

$$
\begin{aligned}
g_{j}^{(k)}= & \frac{2 i}{\sqrt{\omega}}\left\{c g_{1}^{(k)} \rho^{j} \sin j \phi-\left(\alpha g_{n}^{(k)}+\gamma g_{1}^{(k)}\right) \rho^{j-1} \sin (j-1) \phi\right. \\
& \left.+H_{j}^{k} \rho^{j-k} \sin (j-k) \phi-H_{j}^{n}\left(\beta g_{1}^{(k)}+\delta g_{n}^{(k)}\right) \rho^{j-n} \sin (j-n) \phi\right\}
\end{aligned}
$$

for $j \geq 1$. By letting $j=n$ or $j=n+1$,

$$
\begin{aligned}
& A g_{1}^{(k)}+B g_{n}^{(k)}=\rho^{n-k} \sin (n-k) \phi \\
& C g_{1}^{(k)}+D g_{n}^{(k)}=\rho^{n+1-k} \sin (n+1-k) \phi
\end{aligned}
$$

where $A, B, C$ and $D$ are the same as defined in (3.6)-(3.9). If the inverse exists, then $g_{1}^{(k)}$ and $g_{n}^{(k)}$ form a unique solution pair and hence we must have

$$
\Delta=\left|\begin{array}{ll}
A & B \\
C & D
\end{array}\right| \neq 0
$$

or

$$
\begin{aligned}
\Delta= & \rho^{n} \sin \phi(a c \sin (n+1) \phi+(\gamma \delta-\alpha \beta) \sin (n-1) \phi-c \rho(\gamma+\delta) \sin n \phi) \\
& -\left(c \alpha \rho^{2 n}+a \beta\right) \sin ^{2} \phi \neq 0 .
\end{aligned}
$$

Furthermore, if $\Delta \neq 0$, then $g_{1}^{(k)}=\Delta_{1} / \Delta, g_{n}^{(k)}=\Delta_{n} / \Delta$, where

$$
\Delta_{1}=\rho^{n-k} \sin \phi\left(-\alpha \rho^{n} \sin (k-1) \phi+\delta \rho \sin (n-k) \phi-a \sin (n+1-k) \phi\right)
$$

and

$$
\Delta_{n}=\rho^{n-k} \sin \phi\left(-c \rho^{n+1} \sin k \phi+\gamma \rho^{n} \sin (k-1) \phi-\beta \rho \sin (n-k) \phi\right) .
$$

By substituting these into (6.8), we obtain

$$
\begin{aligned}
g_{j}^{(k)}= & \frac{\rho^{n+j-k}}{\Delta} \times\left\{\sin (n-k) \phi\left(\delta \sin j \phi+\frac{\alpha \beta-\gamma \delta}{c \rho} \sin (j-1) \phi\right)\right. \\
& +\sin (n+1-k) \phi(\gamma \sin (j-1) \phi-c \rho \sin j \phi) \\
& \left.+\alpha \rho^{n-1} \sin (j-k) \phi \sin \phi+H_{j}^{k} \frac{\rho^{-n} \Delta}{c \rho \sin \phi} \sin (j-k) \phi\right\},
\end{aligned}
$$

which is (6.2) and (6.3). 
Case II. Suppose that $\omega=0$ so that $\eta_{ \pm}$are two equal roots. From (6.7),

$$
g^{(k)}=\frac{1}{c \rho}\left\{j \rho^{j}\right\}_{j=0}^{\infty} *\left(\bar{c} g_{1}^{(k)}+\hbar^{k}+f^{(k)}\right)
$$

where $\rho=-b / 2 c$. The $j$ th term of $g^{(k)}$ for $j \geq 1$ is now

$$
\begin{aligned}
g_{j}^{(k)}= & \frac{1}{c \rho}\left\{c g_{1}^{(k)} j \rho^{j}-\left(\alpha g_{n}^{(k)}+\gamma g_{1}^{(k)}\right)(j-1) \rho^{j-1}\right. \\
& \left.+H_{j}^{k}(j-k) \rho^{j-k}-H_{j}^{n}\left(\beta g_{1}^{(k)}+\delta g_{n}^{(k)}\right)(j-n) \rho^{j-n}\right\} .
\end{aligned}
$$

In a similar procedure, we may obtain the necessary condition

$$
\Delta=-\alpha c \rho^{2 n}-a \beta+\rho^{n}(a c(n+1)+(\gamma \delta-\alpha \beta)(n-1)-c \rho n(\gamma+\delta)) \neq 0 .
$$

Furthermore, if $\Delta \neq 0$, then $g_{1}^{(k)}=\Delta_{1} / \Delta, g_{n}^{(k)}=\Delta_{n} / \Delta$, where

$$
\Delta_{1}=\rho^{n-k}\left(-\rho^{n} \alpha(k-1)+\rho \delta(n-k)-a(n+1-k)\right)
$$

and

$$
\Delta_{n}=\rho^{n-k}\left(-\rho^{n+1} c k+\rho^{n} \gamma(k-1)-\rho \beta(n-k)\right) .
$$

By substituting these into (6.9), we obtain

$$
\begin{aligned}
g_{j}^{(k)}= & \frac{\rho^{n+j-k}}{\Delta}\left\{(n-k)\left(\delta j+\frac{(\alpha \beta-\gamma \delta)}{c \rho}(j-1)\right)\right. \\
& \left.+(n+1-k)(\gamma(j-1)-c \rho j)+\rho^{n-1} \alpha(j-k)+H_{j}^{k} \frac{\rho^{-n} \Delta}{c \rho}(j-k)\right\},
\end{aligned}
$$

which is (6.5) and (6.6). The proof is complete.

In the case when $\alpha=\beta=\gamma=\delta=0$, if $\sin (n+1) \phi \neq 0$, then the inverse of a typical Toeplitz matrix is given by

$$
g_{j}^{(k)}=\frac{-\rho^{j+1-k}}{a \sin \phi \sin (n+1) \phi} \times \begin{cases}\sin j \phi \sin (n+1-k) \phi, & j<k, \\ \sin k \phi \sin (n+1-j) \phi, & j \geq k,\end{cases}
$$

if $b^{2}-4 a c \neq 0$, and

$$
g_{j}^{(k)}=\frac{-\rho^{j+1-k}}{a(n+1)} \times \begin{cases}j(n+1-k), & j<k \\ k(n+1-j), & j \geq k\end{cases}
$$

if $b^{2}-4 a c=0$. These results agree with the previous works of many authors, in particular that of Haley [5], where (6.10) is expressed in terms of hyperbolic functions when $\eta_{ \pm}$are two distinct real roots. 
In the case when $b=0$, then $b^{2}-4 a c \neq 0$ and $\phi=\pi / 2$. Let

$$
S_{m}=\sin \frac{m \pi}{2}, \quad m \in \mathbb{Z} .
$$

By (6.1), if

$$
\Delta=\rho^{n}(a c+\alpha \beta-\gamma \delta) S_{n+1}-c \rho^{n+1}(\gamma+\delta) S_{n}-a \beta-c \alpha \rho^{2 n} \neq 0,
$$

then (6.2) and (6.3) are simplified to

$$
\begin{aligned}
g_{j}^{(k)}= & \frac{\rho^{n+j-k}}{\Delta}\left\{S_{n-k}\left(\delta S_{j}+\frac{\alpha \beta-\gamma \delta}{c \rho} \cdot S_{j-1}\right)+\alpha \rho^{n-1} S_{j-k}\right. \\
& \left.+S_{n+1-k}\left(\gamma S_{j-1}-c \rho S_{j}\right)\right\}
\end{aligned}
$$

for $j<k$ and

$$
\begin{aligned}
g_{j}^{(k)}= & \frac{\rho^{n+j-k}}{\Delta}\left\{S_{n-j}\left(\delta S_{k}+\frac{\alpha \beta-\gamma \delta}{c \rho} \cdot S_{k-1}\right)+\beta \rho^{1-n} S_{k-j}\right. \\
& \left.+S_{n+1-j}\left(\gamma S_{k-1}-c \rho S_{k}\right)\right\}
\end{aligned}
$$

for $j \geq k$.

Suppose further that $b=0$ and $\alpha=\beta=\gamma=\delta=0$. Then

$$
g_{j}^{(k)}=\frac{-\rho^{j+1-k}}{a S_{n+1}} \times \begin{cases}S_{j} S_{n+1-k}, & j<k, \\ S_{k} S_{n+1-j}, & j \geq k,\end{cases}
$$

if $n$ is even. If $n$ is odd, then the matrix is singular and the inverse does not exist.

\section{Remarks and examples}

The inversion problems of Toeplitz matrices have been studied by many authors $[4,6,7]$. The inversions of Toeplitz matrices with four modified corners have also been studied in [3, 6, 7]. For comparison, we mention briefly the method (adopted from the comments of an anonymous reviewer of the first version of this paper) used in [6]. Let $A$ be the matrix given by (1.1). Let

$$
C=\left(\begin{array}{ccccccc}
b & c & 0 & 0 & \ldots & 0 & 0 \\
a & b & c & 0 & \ldots & 0 & 0 \\
0 & a & b & c & \ldots & 0 & 0 \\
\ldots & \ldots & \ldots & \ldots & \ldots & \ldots & \ldots \\
0 & 0 & 0 & 0 & \ldots & a & b
\end{array}\right)_{n \times n} .
$$

Let $A^{-1}=\left(g_{j k}\right)_{j, k=1}^{n}$ and $C^{-1}=\left(h_{j k}\right)_{j, k=1}^{n}$. We may write

$$
A=C+U S V^{\dagger}
$$


where the dagger denotes transpose,

$$
\begin{gathered}
U=\left(\begin{array}{ll}
e_{1} & e_{n}
\end{array}\right), \quad S=\left(\begin{array}{ll}
\gamma & \alpha \\
\beta & \delta
\end{array}\right), \quad V=\left(\begin{array}{ll}
e_{1}^{\dagger} & e_{n}^{\dagger}
\end{array}\right), \\
e_{1}^{\dagger}=\left(\begin{array}{lllll}
1 & 0 & \cdots & 0 & 0
\end{array}\right)_{1 \times n} \quad \text { and } e_{n}^{\dagger}=\left(\begin{array}{lllll}
0 & 0 & \cdots & 0 & 1
\end{array}\right)_{1 \times n} .
\end{gathered}
$$

If both $C$ and $A$ are invertible, then [6, p. 124]

$$
A^{-1}=C^{-1}+C^{-1} U T V^{\dagger} C^{-1} .
$$

Suppose that $\alpha \beta-\gamma \delta \neq 0$. Then

$$
T^{-1}=-S^{-1}-V^{\dagger} C^{-1} U=\frac{1}{\alpha \beta-\gamma \delta}\left(\begin{array}{cc}
\delta & -\alpha \\
-\beta & \gamma
\end{array}\right)-\left(\begin{array}{ll}
h_{11} & h_{1 n} \\
h_{n 1} & h_{n n}
\end{array}\right) .
$$

Assuming this matrix is invertible, write

$$
T=\left(\begin{array}{ll}
t_{11} & t_{12} \\
t_{21} & t_{22}
\end{array}\right)
$$

The entries of $T$ can be written explicitly in terms of $\alpha, \beta, \gamma, \delta, h_{11}, h_{1 n}, h_{n 1}$ and $h_{n n}$, and

$$
U T V^{\dagger}=\left(\begin{array}{ccccccc}
t_{11} & 0 & 0 & 0 & \ldots & 0 & t_{12} \\
0 & 0 & 0 & 0 & \ldots & 0 & 0 \\
0 & 0 & 0 & 0 & \ldots & 0 & 0 \\
\ldots & \ldots & \ldots & \ldots & \ldots & \ldots & \ldots \\
t_{21} & 0 & 0 & 0 & \ldots & 0 & t_{22}
\end{array}\right)_{n \times n} .
$$

Then by (7.2),

$$
g_{j k}=h_{j k}+h_{j 1}\left(h_{1 k} t_{11}+h_{n k} t_{12}\right)+h_{j n}\left(h_{1 k} t_{21}+h_{n k} t_{22}\right), \quad 1 \leq j, k \leq n .
$$

Hence, given $C^{-1}$, we may find $A^{-1}$. To find $C^{-1}$, suppose that $a c \neq 0$, and let

$$
\eta_{ \pm}=\frac{-b \pm \sqrt{b^{2}-4 a c}}{2 a}
$$

be the two roots of $a z^{2}+b z+c=0$. The first row and column of $C^{-1}$ can be found by solving the boundary problems

$$
c h_{1, k-1}+b h_{1 k}+a h_{1, k+1}=0, \quad 1 \leq k \leq n, h_{10}=-1 / c, h_{1, n+1}=0,
$$

and

$$
a h_{j-1,1}+b h_{j 1}+c h_{j+1,1}=0, \quad 1 \leq j \leq n, h_{01}=-1 / a, h_{n+1,1}=0 .
$$

If $b^{2}-4 a c \neq 0$, then

$$
h_{1 k}=\frac{\eta_{-}^{n+1} \eta_{+}^{k}-\eta_{+}^{n+1} \eta_{-}^{k}}{c\left(\eta_{+}^{n+1}-\eta_{-}^{n+1}\right)}, \quad 1 \leq k \leq n
$$


and

$$
h_{j 1}=\frac{\eta_{+}^{n+1-j}-\eta_{-}^{n+1-j}}{a\left(\eta_{-}^{n+1}-\eta_{+}^{n+1}\right)}, \quad 1 \leq j \leq n .
$$

If $b^{2}-4 a c=0$, then

$$
h_{1 k}=\frac{(k-n-1) \eta^{k}}{c(n+1)}, \quad 1 \leq k \leq n,
$$

and

$$
h_{j 1}=\frac{(j-n-1) \eta^{-j}}{a(n+1)}, \quad 1 \leq j \leq n .
$$

Given the first row and column of $C^{-1}$, the remaining entries can be obtained recursively by Trench's recursion [7, p. 207] or explicitly from the Gohberg-Semencul formula [4]. Note that the last two equations are just (6.11) when $j=1$ or $k=1$, while (6.10) is much simpler than the two formulae for $b^{2}-4 a c \neq 0$ given above.

The method mentioned here seems to be elegant; however, beside the restriction of $\alpha \beta-\gamma \delta \neq 0$, computation may be extremely tedious even for a simple Toeplitz matrix. We will justify our statement by two examples given below. Besides, explicit formulae, instead of algorithms, are the main concern in this study.

We also note that evaluating $\phi$ and the trigonometric functions may be avoided by applying the Chebyshev polynomials of the first and second kinds as suggested by Dow in [3].

EXAmple 1. Suppose that $n=4, a=3, b=2, c=1, \alpha=1, \beta=\gamma=-1, \delta=0$ in matrix $A_{n}$. Then $\rho=\sqrt{3}, \alpha \beta-\gamma \delta=-1 \neq 0$, and $\cos \phi=-1 / \sqrt{3}$, so that $\sin \phi=\sqrt{2} / \sqrt{3}, \sin 2 \phi=-2 \sqrt{2} / 3, \sin 3 \phi=\sqrt{2} / 3 \sqrt{3}, \quad \sin 4 \phi=4 \sqrt{2} / 9$ and $\sin 5 \phi=-11 \sqrt{2} / 9 \sqrt{3}$. Since

$$
\begin{aligned}
\Delta= & 9 \cdot\left(\frac{\sqrt{2}}{\sqrt{3}}\right)\left(3 \cdot\left(\frac{-11 \sqrt{2}}{9 \sqrt{3}}\right)+\frac{\sqrt{2}}{3 \sqrt{3}}+\sqrt{3} \cdot\left(\frac{4 \sqrt{2}}{9}\right)\right) \\
& -(81-3) \cdot\left(\frac{2}{3}\right)=-64 \neq 0,
\end{aligned}
$$

by (6.2),

$$
g_{j}^{(k)}=\frac{1}{-64} \times(\sqrt{3})^{4+j-k} \times c_{j k},
$$

where

$$
\begin{aligned}
c_{j k}= & \frac{-1}{\sqrt{3}} \sin (4-k) \phi \sin (j-1) \phi-\sin (5-k)(\sin (j-1) \phi+\sqrt{3} \sin j \phi) \\
& +3 \sqrt{2} \sin (j-k) \phi
\end{aligned}
$$

for $j<k$ and

$$
\begin{aligned}
c_{j k}= & \frac{-1}{\sqrt{3}} \sin (4-j) \phi \sin (k-1) \phi-\sin (5-j)(\sin (k-1) \phi+\sqrt{3} \sin k \phi) \\
& -\frac{\sqrt{2}}{9} \sin (k-j) \phi
\end{aligned}
$$


for $j \geq k$. This yields

$$
(\sqrt{3})^{4+j-k} \times\left(c_{j k}\right)_{4 \times 4}=\left(\begin{array}{cccc}
-8 & -20 & 16 & -4 \\
-4 & 6 & -24 & 14 \\
32 & -16 & 0 & -16 \\
-52 & 14 & 8 & -10
\end{array}\right)
$$

and

$$
\left(\begin{array}{llll}
1 & 1 & 0 & 1 \\
3 & 2 & 1 & 0 \\
0 & 3 & 2 & 1 \\
-1 & 0 & 3 & 2
\end{array}\right)^{-1}=\frac{1}{32}\left(\begin{array}{cccc}
4 & 10 & -8 & 2 \\
2 & -3 & 12 & -7 \\
-16 & 8 & 0 & 8 \\
26 & -7 & -4 & 5
\end{array}\right)
$$

For comparison, we note that since

$$
\eta_{ \pm}=\frac{-2 \pm \sqrt{4-12}}{6}=-\frac{1}{3} \pm i \frac{\sqrt{2}}{3}
$$

Equation (7.4) is

$$
h_{1 k}=\frac{\left(-\frac{1}{3}-i \frac{\sqrt{2}}{3}\right)^{5}\left(-\frac{1}{3}+i \frac{\sqrt{2}}{3}\right)^{k}-\left(-\frac{1}{3}+i \frac{\sqrt{2}}{3}\right)^{5}\left(-\frac{1}{3}-i \frac{\sqrt{2}}{3}\right)^{k}}{\left(-\frac{1}{3}+i \frac{\sqrt{2}}{3}\right)^{5}-\left(-\frac{1}{3}-i \frac{\sqrt{2}}{3}\right)^{5}},
$$

which is more complicated and obviously leads us to tedious follow-up computations.

In the case when $b=0$, the evaluation becomes much simpler due to the fact that $S_{k}=-1,0,1$, and $S_{k \pm 1}=0$ if $S_{k} \neq 0$. We illustrate this by the following example.

ExAmple 2. Suppose that $n=6, a=c=1, b=0, \alpha=\delta=1$ and $\beta=\gamma=-1$ in matrix $A_{n}$. We have $\rho=1, \alpha \beta-\gamma \delta=0$ and, by (6.12),

$$
\Delta=a c S_{7}-a \beta-c \alpha=-1+1-1=-1,
$$

the inverse exists, and by (6.13) and (6.14) of Theorem 6.1,

$$
g_{j}^{(k)}=- \begin{cases}S_{6-k}\left(S_{j}\right)+S_{j-k}+S_{7-k}\left(-S_{j-1}-S_{j}\right), & j<k \\ S_{6-j}\left(S_{k}\right)-S_{k-j}+S_{7-j}\left(-S_{k-1}-S_{k}\right), & j \geq k\end{cases}
$$

which gives

$$
\left(\begin{array}{llllll}
-1 & 1 & 0 & 0 & 0 & 1 \\
1 & 0 & 1 & 0 & 0 & 0 \\
0 & 1 & 0 & 1 & 0 & 0 \\
0 & 0 & 1 & 0 & 1 & 0 \\
0 & 0 & 0 & 1 & 0 & 1 \\
-1 & 0 & 0 & 0 & 1 & 1
\end{array}\right)^{-1}=\left(\begin{array}{cccccc}
-1 & 2 & 1 & -2 & -1 & 2 \\
0 & 1 & 1 & -1 & -1 & 1 \\
1 & -1 & -1 & 2 & 1 & -2 \\
0 & -1 & 0 & 1 & 1 & -1 \\
-1 & 1 & 1 & -1 & -1 & 2 \\
0 & 1 & 0 & -1 & 0 & 1
\end{array}\right) .
$$

We note that, since $\alpha \beta-\gamma \delta=0$, the method used in [6] as mentioned above is not applicable. 
It is also interesting to note that there exist singular matrices which may be invertible when the corner or corners are perturbed. In these cases, the method used in [6] is still not applicable (which demands that $C$ in (7.1) is invertible). The following is an example of such a matrix that is solved by Theorem 6.1 of this paper.

EXAmple 3. Suppose that $n=5, a=c=1, b=-1, \alpha=0$, and $\beta=\delta=-1, \gamma=1$ in matrix $A_{n}$. We have $\rho=1, \alpha \beta-\gamma \delta=1, \gamma+\delta=0$ and $\cos \phi=1 / 2$, so that $\sin \phi=\sin 2 \phi=\sqrt{3} / 2, \sin 3 \phi=\sin 6 \phi=0, \sin 4 \phi=\sin 5 \phi=-\sqrt{3} / 2$. By (6.1) $\Delta=3 / 2$, the inverse exists, and by (6.2) and (6.3) of Theorem 6.1,

$$
g_{j}^{(k)}=\frac{1}{\Delta} \times \begin{cases}(\sin (5-k) \phi+\sin (6-k) \phi)(\sin (j-1) \phi-\sin j \phi), & j<k, \\ (\sin (5-j) \phi+\sin (6-j) \phi)(\sin (k-1) \phi-\sin k \phi) & \\ -\frac{\sqrt{3}}{2} \sin (k-j), & j \geq k\end{cases}
$$

This yields

$$
\left(\begin{array}{lllll}
0 & 1 & 0 & 0 & 0 \\
1 & -1 & 1 & 0 & 0 \\
0 & 1 & -1 & 1 & 0 \\
0 & 0 & 1 & -1 & 1 \\
-1 & 0 & 0 & 1 & -2
\end{array}\right)^{-1}=\frac{1}{2}\left(\begin{array}{ccccc}
2 & 1 & -1 & -2 & -1 \\
2 & 0 & 0 & 0 & 0 \\
0 & 1 & 1 & 2 & 1 \\
-2 & 1 & 3 & 2 & 1 \\
-2 & 0 & 2 & 2 & 0
\end{array}\right)
$$

\section{References}

[1] S. S. Cheng, Partial difference equations (Taylor and Francis, London, 2003).

[2] S. S. Cheng and L. Y. Hsieh, "Inverses of matrices arising from difference operators", Util. Math. 38 (1990) 65-77.

[3] M. Dow, "Explicit inverses of Toeplitz and associated matrices", ANZIAM J. 44 (2003) 185-215.

[4] I. C. Gohberg and A. A. Semencul, "On the inversion of finite Toeplitz matrices and their continuous analogs", Mat. Issled 7 (1972) 201-223 (in Russian).

[5] S. B. Haley, "Solutions of band matrix equations by projection-recurrence", Linear Algebra Appl. 32 (1980) 33-48.

[6] A. S. Householder, The theory of matrices in numerical analysis (Dover Publications, New York, 1975).

[7] W. F. Trench, "An algorithm for the inversion of finite Toeplitz matrices", SIAM J. Appl. Math. 12 (1964) 515-522.

[8] W. C. Yueh, "Eigenvalues of several tridiagonal matrices", Appl. Math. E-Notes 5 (2005) 66-74.

[9] W. C. Yueh, "Explicit inverses of several tridiagonal matrices", Appl. Math. E-Notes 6 (2006) 74-83.

[10] W. C. Yueh and S. S. Cheng, "Explicit eigenvalues and inverses of several Toeplitz matrices", ANZIAM J. 48 (2006) 73-97. 